\title{
Inhomogeneous soliton ratchets under two ac forces
}

\author{
Luis Morales-Molina \\ Max-Planck Institut für Physik Komplexer Systeme, Nöthnitzer Strasse 38, 01187 Dresden, Germany
}

Franz G. Mertens*

Physikalisches Institut, Universität Bayreuth, D-85440 Bayreuth, Germany

\begin{abstract}
Angel Sánchez ${ }^{\dagger}$
Grupo Interdisciplinar de Sistemas Complejos (GISC) and Departamento de Matemáticas, Universidad Carlos III de Madrid, Avenida de la Universidad 30, 28911 Leganés, Madrid, Spain, and Instituto de Biocomputación y Física de Sistemas Complejos (BIFI), Facultad de Ciencias, Universidad de Zaragoza, 50009 Zaragoza, Spain
\end{abstract}

(Received 15 August 2005; published 10 April 2006)

\begin{abstract}
We extend our previous work on soliton ratchet devices [L. Morales-Molina et al., Eur. Phys. J. B 37, 79 (2004)] to consider the joint effect of two ac forces including nonharmonic drivings, as proposed for particle ratchets by Savele'v et al. [Europhys. Lett. 67, 179 (2004); Phys. Rev. E 70, 066109 (2004)]. Current reversals due to the interplay between the phases, frequencies, and amplitudes of the harmonics are obtained. An analysis of the effect of the damping coefficient on the dynamics is presented. We show that solitons give rise to nontrivial differences in the phenomenology reported for particle systems that arise from their extended character. A comparison with soliton ratchets in homogeneous systems with biharmonic forces is also presented. This ratchet device may be an ideal candidate for Josephson junction ratchets with intrinsic large damping.
\end{abstract}

DOI: 10.1103/PhysRevE.73.046605

PACS number(s): 05.45.Yv, 85.25.Cp, 73.40.Ei, 87.16.Nn

The understanding of ratchet mechanisms is a very active field of wide interest by its potential application in the design of devices with new transport properties. The key feature of ratchet devices is their ability to rectify the motion of particles subjected to an external ac force with zero time average. Originally proposed as a toy model for molecular motors, in the last decade many proposals have been put forward for devices that use this ratchet phenomenon in different applications [1]. Ratchets working with extended particles (solitons) were subsequently studied as a generalization of particle ratchets [2]. Recently, an overdamped ratchet device for a single particle driven by two ac forces was introduced by Savele'v and coworkers [3], in which the combination of the two drivings produced a variety of interesting phenomena and allowed a finely tunable control. In view of the rich behavior demonstrated by this system, a very natural issue is its extension to soliton ratchets that have important applications which can benefit from this proposal.

Soliton ratchets under the presence of two ac forces have been extensively studied [4,5] in homogeneous systems, i.e., without an underlying ratchet potential. In this case, it has been shown that the ratchet mechanism works only for asymmetric biharmonic forces, where the appearance of a directed translational motion is a result of the effective coupling between the internal mode (oscillations of the soliton width) and the external driving force. In this paper, we focus on a soliton ratchet device recently studied by us $[7,8]$, based on a nonlinear Klein-Gordon model with a lattice of deltalike in-

\footnotetext{
*Electronic address: Franz.Mertens@uni-bayreuth.de

${ }^{\dagger}$ URL: http://gisc.uc3m.es/ anxo
}

homogeneities that induce a ratchet potential for the solitons. As we will see, one of the advantages of our model as compared to the homogeneous system is that it works irrespectively of the symmetry of the ac driving: Directional transport takes place for ac forces with commensurate frequencies. Moreover, in the homogeneous system the motion drastically decreases for increasing damping, due to the slowing down of the soliton width oscillations [5], while in the present system the ratchet phenomenon is present up to rather large values for the damping.

Our model, first introduced in [7], is given by

$$
\phi_{t t}+\beta \phi_{t}-\phi_{x x}+\sin (\phi)[1+V(x)]=f(t),
$$

where $f(t)=A_{1} \sin \left(\omega_{1} t+\theta_{1}\right)+A_{2} \sin \left(\omega_{2} t+\theta_{2}\right)$, being $A_{1}, A_{2}$ the respective amplitudes of forces, $\omega_{1}, \omega_{2}$ the frequencies, and $\theta_{1}$ and $\theta_{2}$ the phases of the harmonics (see, e.g., [3]). Here we focus on the sine-Gordon model, although the same scheme can also be applied to the general nonlinear KleinGordon model [8]. For $V(x)$, we choose a spatially periodic potential, where the unit cell is given by an asymmetric array of delta functions (inhomogeneities) in order to produce a ratchetlike phenomenon. Specifically, the unit cell, of length $L$, is defined by three inhomogeneities with the same intensity, the first one located at the beginning of the cell, the second one at a distance $a$ from the first one, and the third one at a distance $b$ from the second one, i.e.,

$$
V(x)=\epsilon \sum_{n}\left[\delta\left(x-x_{1}-n L\right)+\delta\left(x-x_{2}-n L\right)+\delta\left(x-x_{3}-n L\right)\right],
$$

where $L=a+b+c, a=x_{2}-x_{1}, b=x_{3}-x_{2}$, and $c=L+x_{1}-x_{3}$, with $x_{1}<x_{2}<x_{3}$. The parameters $(a, b, c)$ are chosen to be 
comparable to the static soliton width $l_{0}$ in absence of inhomogeneities. In addition, these should fulfill the conditions $a, b<c$ with $a \neq b$.

As a preliminary step, we begin our study by analyzing how the system works in different regimes of damping. This is very relevant to applications such as Josephson junctions. While the standard Josephson junctions work usually at small damping, junctions with intrinsically high damping such as superconductor insulator normal-conductor insulator superconductor (LJJ) or high- $T_{c}$ LJJ technology can also be fabricated [6]. For this particular aspect, we look at the case of a single harmonic component in Eq. (1) $\left(A_{1}=A, \omega_{1}\right.$ $\left.=\omega ; A_{2}=0, \omega_{2}=0\right)$. In this case, according to $[7,8]$ we have a directed motion whose direction is determined by the position of the inhomogeneities, and the dynamics reduces to a system very much like a rocking ratchet for a single particle [9]. Our results are shown in Fig. 1, where we have chosen a lattice of inhomogeneities whose configuration $(a<b)$ yields a negative current [7]. Hereafter $\langle\dot{X}\rangle$ means the time average of the velocity. The figure demonstrates the increment of the efficiency upon decreasing the damping $\beta$. One can note the increment of the number of windows of motion as well as the absolute value of the mean velocity [Figs. 1(a) and 1(b)]. On the other hand, one can observe a shift of the windows to lower values of the force amplitude as the damping decreases. The behavior for a particular value of the force amplitude as a function of the damping is shown in Fig. 1(c).

We note also that for these frequencies with lower damping values, the dynamics results depend on the initial conditions, which may lead to a chaotic dynamics.

Let us now move to the situation with two simultaneously acting ac forces. For the time being, we work with sinusoidal forces as in Eq. (1), and we will consider later the case of rectangular pulses as in [3]. With two harmonics present (doubly rocked ratchet) we have a system in which the symmetry can be broken both spatially [reflection symmetry $V(x)=V(-x)$ ] and temporally [time shift symmetry for $f(t)[4,5]$ with $\left.T=2 \pi / \omega_{1}\right]$. As we will now see, it is possible to obtain current reversals irrespective of the symmetry of the biharmonic force. The reason is that the underlying mechanism of transport studied for homogeneous systems subjected to biharmonic forces that required temporal symmetry breaking $[4,5]$ is not responsible for the directional transport in our present model, as can be inferred from the fact that in the inhomogeneous system we have directed transport under large damping. We examine the dynamics of Eq. (1) in the partially adiabatic regime $\omega_{1} / \omega_{2} \ll 1$ with $\omega_{1} \ll \omega_{0} \quad\left(\omega_{0}=0.5\right.$ is the half of the lowest phonon $)$ and $\omega_{2}<\omega_{0}$, setting $A_{1}=A_{2}=A$. In the partially adiabatic regime, multiple current reversals are possible in the particle ratchet system [3]. Figure 2 confirms clearly the existence of several current reversals in our soliton device. The results show that we can reverse the direction of motion by changing the force amplitudes, which, as in the particle case, opens the possibility to control the rectification properties in great detail.

In the previous analysis we have taken $\beta=1$, i.e., a rather large damping where the inertial effects are small. However, from the above discussion for one harmonic, we expect changes in the behavior also in the case of a biharmonic
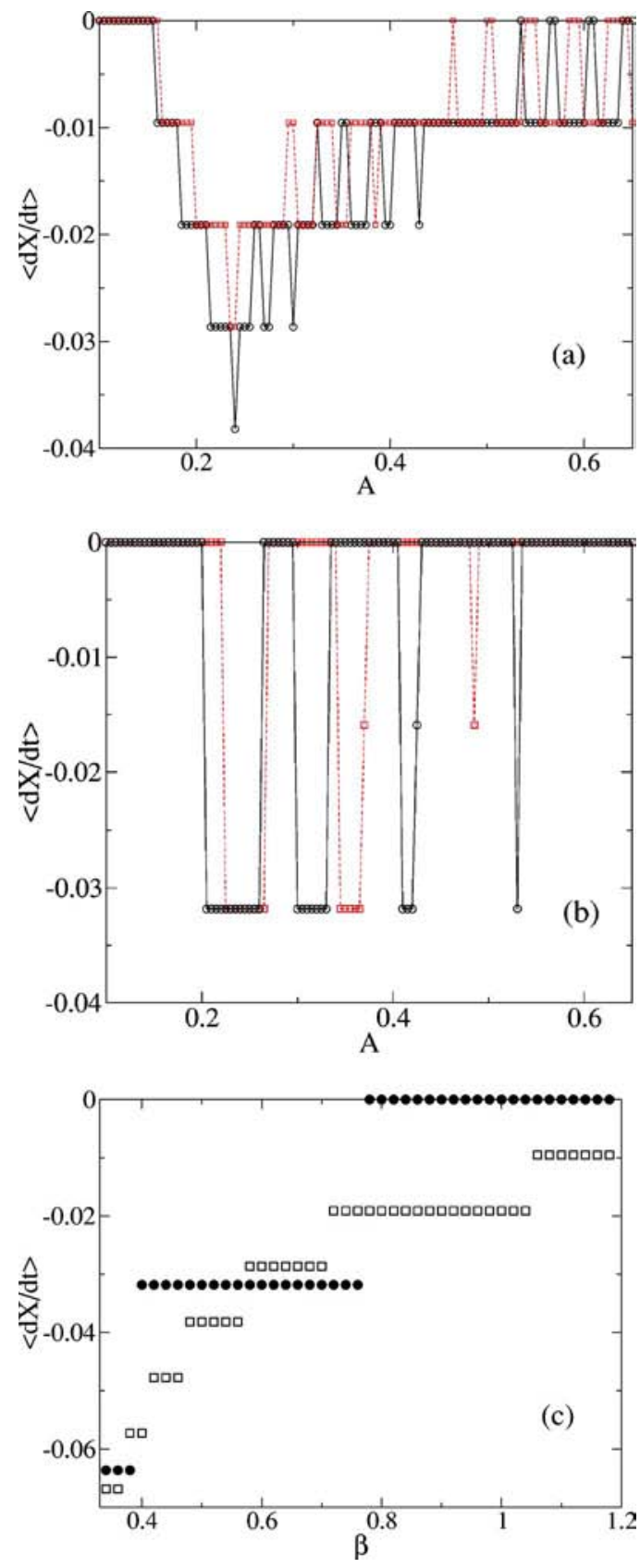

FIG. 1. (Color online) (a) and (b) Mean velocity versus force amplitude for two frequencies (a) $\omega=0.015$ and (b) $\omega=0.05$. In both cases, results for two damping values $\beta=0.8$ (circles, solid line) and $\beta=1$ (squares, dashed line) are shown. Lines are only guides to the eye. (c) Mean velocity versus damping coefficient $\beta$ for two different frequencies with $A=0.2$. squares: $\omega=0.015$; filled circles: $\omega=0.05$. The parameters used in all panels are $\epsilon=0.5, x_{1}=0.5$, $x_{2}=1, x_{3}=2.3$, and $L=4$.

force when the damping is reduced. Figure 3 exhibits indeed a different picture for the dynamics as compared with Fig. 2. The main differences are related to the shift of the windows of motion toward lower force amplitude values as well as an increase of the absolute value of the mean velocity for some force amplitudes. Interestingly, we note from this picture a nontypical behavior for the average velocity close to the region where the first current reversal takes place: We observe 


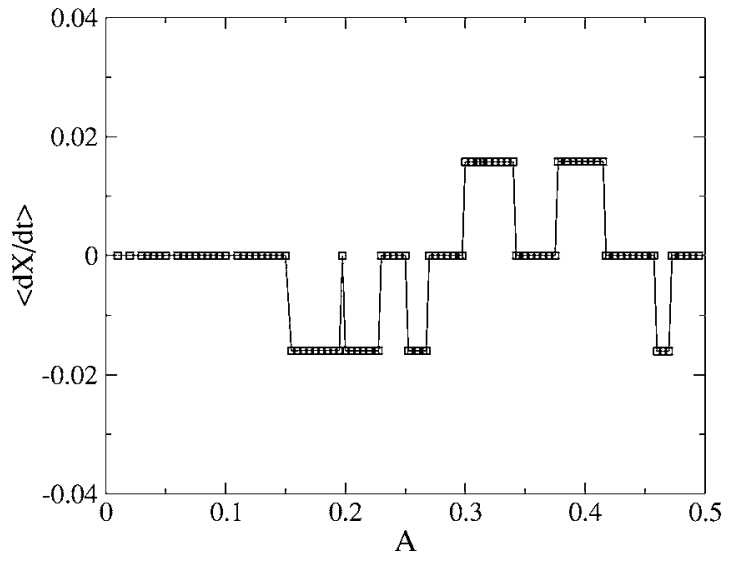

FIG. 2. Mean velocity versus force amplitude $A_{1}=A_{2}=A$ simulations of Eq. (1); the parameters used are $\beta=1, \omega_{1}=0.025$, $\omega_{2}=0.3, \epsilon=0.5, \theta_{1}=\theta_{2}=0, x_{1}=0.5, x_{2}=1, x_{3}=2.3$, and $L=4$. The solid line is a guide for the eye.

that while for some values of the force amplitude the windows of motion are suppressed; for other cases, the absolute value of the average velocity is enhanced and even reversed.

This issue can be further examined in Fig. 4, where the dependence of the average velocity on the relative phase of the drivings is plotted. Here we see that by changing the phases we reverse the direction of motion. This behavior is not only restricted to this singular region in Fig. 3; in fact, it can be observed close to the regions where the currents are reversed in Fig. 3. We stress that we have taken $\theta_{1}=0$ and $\theta_{2}=\theta$ without loss of generality, since although the dynamics obviously changes with both phases $\theta_{1}$ and $\theta_{2}$, one can always map the choice for $\theta_{1}$ and $\theta_{2}$ into the previous representation through the transformations $\theta_{2}^{\prime}=\theta_{2}-\left(\omega_{2} / \omega_{1}\right) \theta_{1}$ with $\theta_{1}^{\prime}=0$. A feature of the behavior for this doubly rocked ratchet system, which differs from the homogeneous system case, is the quantization of the velocity dependence on the phase (see Fig. 4 and compare with Fig. 2 in [5]). This is yet another evidence that the ratchet mechanisms at work in the homogeneous and inhomogeneous cases are not the same. Nevertheless, despite this quantized nature, it is feasible to

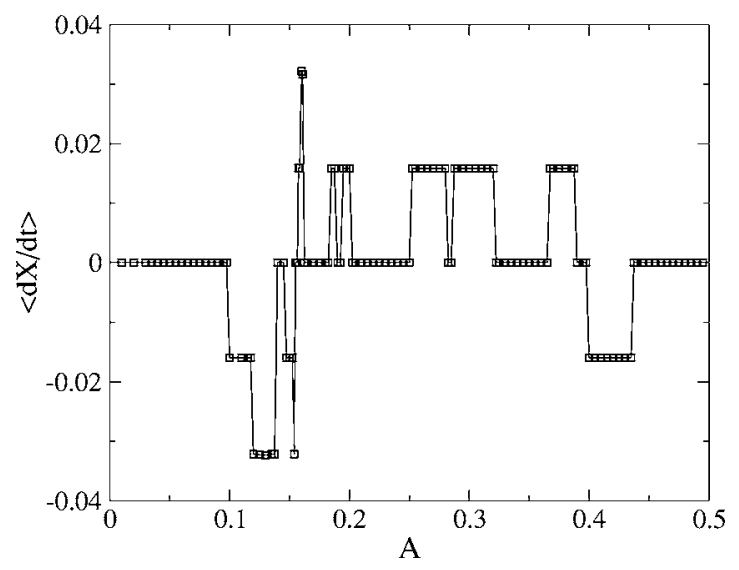

FIG. 3. Mean velocity versus force amplitude. $\beta=0.4, A_{1}=A_{2}$ $=A$ simulations of Eq. (1). The other parameters are the same as in Fig. 2. The solid line is a guide for the eye.

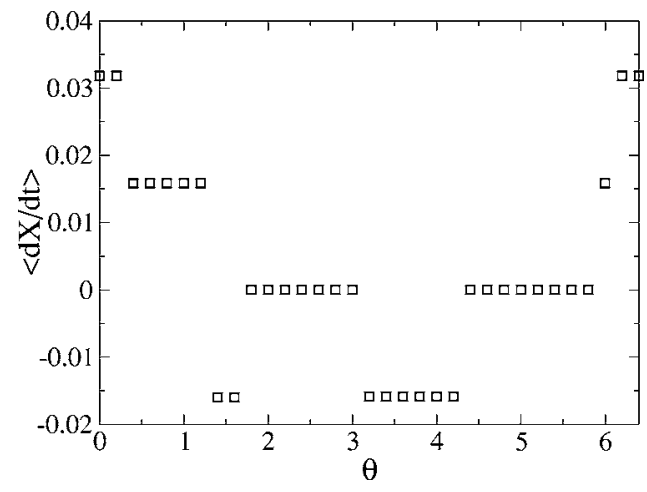

FIG. 4. Mean velocity versus $\theta$. Here we have taken $\theta_{1}=0$ and $\theta_{2}=\theta$ in Eq. (1). The other parameters are $A_{1}=A_{2}=0.16, \beta=0.4$, $\omega_{1}=0.025$, and $\omega_{2}=0.3$.

control the direction of motion by tuning the phases of the biharmonic force as in the homogeneous case.

In view of the similarities with the single particle ratchet system in [3], it is important to assess the degree of similarity between the two systems. Savele'v and coworkers focus mainly on the case of rectangular wave signals as the asymmetry and nonlinearity-induced mixing are separable [3]. For such rectangular wave signals in the fully adiabatic regime with a sawtooth ratchet potential, Savele'v et al. report changes in the dynamics only for a relation between the frequencies given by $\omega_{2} / \omega_{1}=(2 m-1) /(2 n-1)$. As Fig. 5 shows, in our case with $\omega_{1}, \omega_{2} \ll \omega_{0}$ the behavior turns out to be much more complicated. The average velocity fluctuates around the value indicated by a horizontal line, but it does exhibit very many peaks. Most importantly, unlike the single particle picture shown in [3], here the peaks appear not only for fractional harmonics $\omega_{2} / \omega_{1}=(2 m-1) /(2 n-1)$, but also for harmonics that fulfill the relations $\omega_{2} / \omega_{1}=(2 m-1) / 2 n$ and $\omega_{2} / \omega_{1}=2 m /(2 n-1)$.

The reason for the difference between the particle and the soliton doubly rocked ratchets can be traced back to the extended character of our solitons. It is well known that the soliton dynamics is affected by the deformation of the soliton width that accompanies its motion along an array of inhomogeneities [8]. This in turn modifies the effective potential arising in the description of the soliton as point particle, as well as in the variations of the corresponding effective force. Accordingly, there is a large degree of feedback between the soliton width and the soliton motion. To obtain some insight on these issues, it is necessary to study the evolution of the degrees of freedom that are involved. In order to do so, we resort to the use of the collective coordinate approach [10] which involves the soliton width as an additional degree of freedom. In doing so, we find that the soliton can be described by two collective variables $X$ and $l$, whose expressions are given by Eqs. (4) and (5) in [8], with the difference that now $f(t)$ contains the two rectangular wave signals whose expression appears in the caption of Fig. 5. This is in contrast to the single particle doubly rocked ratchet of Savele'v et al., because of the appearance of the width degree of freedom. This collective coordinate approach explains in full detail the results of the simulations, as shown in Fig. 5 for the fully 

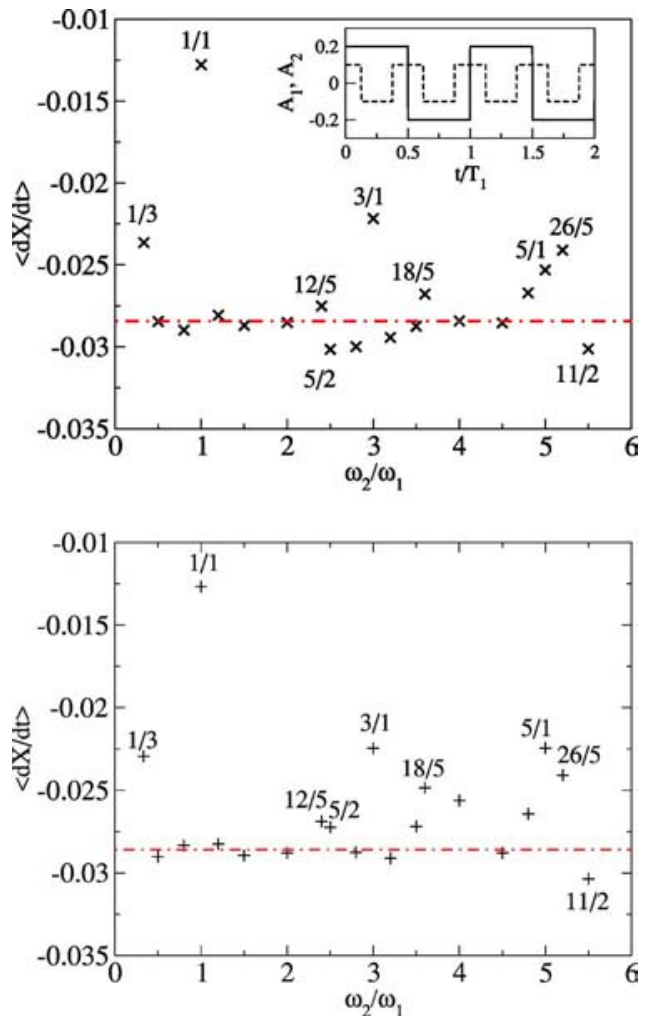

FIG. 5. (Color online) Mean velocity for different frequency ratios $\omega_{2} / \omega_{1}$ in the fully adiabatic regime with rectangular wave signals $f(t)=A_{1}(t)+A_{2}(t)$. The parameters are $\omega_{1}=0.005$, $A_{1}=0.25, A_{2}=0.05, \beta=1$, and $\theta_{1}=\theta_{2}=0$. Top panel: simulations. Bottom panel: collective coordinates. Inset of the top panel: rectangular wave signals. Bottom: collective coordinates. $A_{1}(t)=A_{1} \operatorname{sgn}\left[\sin \left(\omega_{1} t+\theta_{1}\right)\right] \quad($ solid line $) ; \quad A_{2}(t)=A_{2} \operatorname{sgn}\left[\sin \left(\omega_{2} t\right.\right.$ $\left.\left.+\theta_{2}\right)\right]$ (dashed line); $A_{2}=A_{1} / 2=0.1, \omega_{2}=2 \omega_{1}, \theta_{1}=0$, and $\theta_{2}=\pi / 2$.

adiabatic regime. Again, as in the simulations of Eq. (1) we note not only the existence of peaks for the frequency ratios $\omega_{2} / \omega_{1}=(2 m-1) /(2 n-1)$, but also for some frequencies ratio that fulfill the relation $\omega_{2} / \omega_{1}=(2 m-1) / 2 n$ and $\omega_{2} / \omega_{1}=2 m /(2 n-1)$, absent in the single particle picture [3]. This result is a clear demonstration of the role of the soliton width in the dynamics.
To conclude, we have generalized the results obtained for doubly rocked particle ratchets [3] to extended systems, finding phenomena that arise from the intrinsic width of the solitons. It was shown that the direction of motion can be modified by changing the relation between the frequencies ratio, the phases of the harmonic forces, as well as their amplitudes. However, in the frame of the fully adiabatic regime of our soliton ratchet we find many more peaks than in the particle ratchet system of Savele'v et al., as we see special velocities for several types of frequency ratios. We have been able to show that this is thoroughly accounted for by the interplay of the soliton width and motion. We have also compared to doubly rocked soliton ratchets in homogeneous systems $[4,5]$ and verified that, although the soliton width is involved in both cases, the mechanism for the appearance of the ratchet effect is different. Aside from the fact that homogeneous soliton ratchets arise only for asymmetric biharmonic drivings, further important differences include the damping dependence of the velocity and the quantization of the dependence of the velocity on the relative phase. We emphasize that our ratchet system can be straightforwardly implemented in a Josephson junction device [6]. In that case, the very many possibilities for the motion we have reported here would allow for a highly controllable device that can be tailored to fit different specific applications. The property that the ratchet phenomenon is observed even in the presence of large damping makes this system preferable to a homogeneous one driven by a biharmonic force, and makes it much more suitable for real life applications. Finally, we note that an analysis similar to the present one can be extended to incommensurate ac forces with irrational values for the relation $\omega_{2} / \omega_{1}$. However, our preliminary results show that this choice gives rise to phenomena that require careful attention, and therefore it will be the object of a future investigation.

L.M.M. thanks B. Lindner for comments. This work has been supported by the Ministerio de Ciencia y Tecnología of Spain through Grants No. BFM2003-07749C05-01, No. FIS2004-01001, and No. NAN2004-09087C03-03 (A.S.) and by "Acciones Integradas HispanoAlemanas" HA2004-0034-D/04/39957 and by the Junta Andalucía under the Project No. FQN-0207.
[1] P. Hänggi and R. Bartussek, Lecture Notes in Physics, Vol. 476 (Springer, Berlin, Heidelberg, 1996), p. 294; F. Jülicher, A. Ajdar, and J. Prost, Rev. Mod. Phys. 69, 1269 (1997); P. Reimann, Phys. Rep. 361, 57 (2002); H. Linke, Appl. Phys. A: Mater. Sci. Process. 75, 167 (2002).

[2] O. M. Braun and Yu. S. Kivshar, The Frenkel-Kontorova Model (Springer, Berlin, 2004).

[3] S. Savel'ev, F. Marchesoni, P. Hänggi, and F. Nori, Europhys. Lett. 67, 179 (2004); Phys. Rev. E 70, 066109 (2004).

[4] S. Flach, Y. Zolotaryuk, A. E. Miroshnichenko, and M. V. Fistul, Phys. Rev. Lett. 88, 184101 (2002). M. Salerno and Y. Zolotaryuk, Phys. Rev. E 65, 056603 (2002).
[5] L. Morales-Molina, N. R. Quintero, F. G. Mertens, and A. Sánchez, Phys. Rev. Lett. 91, 234102 (2003).

[6] A. Sterck, S. Weiss, and D. Koelle, Appl. Phys. A: Mater. Sci. Process. 75, 253 (2002); D. Koelle, R. Kleiner, F. Ludwig, E. Dantsker, and J. Clarke, Rev. Mod. Phys. 71, 631 (1999).

[7] L. Morales-Molina, F. G. Mertens, and A. Sánchez, Eur. Phys. J. B 37, 79 (2004).

[8] L. Morales-Molina, F. G. Mertens, and A. Sánchez, Phys. Rev. E 72, 016612 (2005).

[9] R. Bartussek, P. Hänggi, and J. G. Kissner, Europhys. Lett. 28, 459 (1994).

[10] A. Sánchez and A. R. Bishop, SIAM Rev. 40, 579 (1998). 